\title{
Influence of Gas and Liquid Viscosity on Turbine and Positive Displacement Meters Calibration
}

\author{
Pedišius Nerijusa ${ }^{a}$ Zygmantas Gediminas and Maslauskas Eugenijus \\ Lithuanian Energy Institute, Breslaujos str. 3 LT-44403 Kaunas, Lithuania
}

\begin{abstract}
Résumé. L'article présente une étude de l'impact de la viscosité des liquides et des gaz sur les erreurs de mesure des débitmètresrotatifs à palette et à turbine. Deux débitmètres rotatifs à palette ont été étudiés en laboratoire et en conditions réelles dans les flux de quatre fluides de viscosité différente $v=(0,73-4,6) \mathrm{mm}^{2} / \mathrm{s}$, ainsi que quatre types dedébitmètres à turbine dans un flux d'air (gaz) de pression atmosphérique ou augmentée $\left(v=(0,44-2,74) \mathrm{mm}^{2} / \mathrm{s}\right)$. Les variations d'erreurs des débitmètres ont été analysées en utilisant le critère de similitude Re.

Les deux intervalles de variation des erreurs des débitmètres, qui diffèrent par leur valeur d'erreur maximale, ont été distinguésen fonction deRe. Avant cette valeur d'erreur maximale, lorsque le débit augmente, l'impact de la friction mécanique et de la viscosité diminue graduellement,un déplacement est observé des erreurs vers des valeurs positives plus grandes jusqu'à ce que le débitatteigne le régime de turbulence. Le changement des erreurs selon la loi universelle de dépendance deRevers des valeurs négatives plus grandeset le déplacement des erreursvers des valeurs asymptotiques est typiqueau régime de débit de turbulence. L'article traiteles raisons de ces régularités. Il montre également que, malgré des différents principes de fonctionnement des débitmètres à pale rotatifs et à turbine, la dépendance des erreursdeReest similaire et une prévision de variations des erreurs après un changement des conditions du flux est possible.
\end{abstract}

\section{Introduction}

Regardless of development and application of new measurement methods, mechanical meters with turning rotors are widely utilized in liquid and gas volume and flow rate measurement due to their accuracy and reliability.

Positive displacement meters with sliding vanes, often called rotary vane meters, occupy a special place in the field of liquid fuel flow measurements. Their measurement errors are determined by liquid flow leakage through the gaps between the sliding rotor vanes, which comprise separate chambers, and the casing. The main disadvantage of these meters is their great sensitivity to liquid flow regime and to the change of the liquids' density and viscosity because of their type, temperature and pressure.

Turbine meters are widely utilized for measurements of high flow rates of compressed natural gas. However, for a long time they had been calibrated for atmospheric pressure although they were used for measurements of highly compressed gas flowing through gas pipes. Growth of gas density under increasing pressure significantly decreases gas kinematic viscosity and results in difficulties when extrapolating results of calibration at the laboratory to operating conditions.
Complex and expensive procedures and equipment are necessary for calibration and to ensure stable permanent measurement accuracy of both type meters in operating conditions. Thus, it is especially relevant to investigate and determine measurement accuracy of meters under the variation of flow regime and physical properties of flows.

Performance of these meters has been investigated rather widely. Despite this fact, increasing demands for measurement accuracy, taking into account the type of fluids and operation conditions, require deeper knowledge on influencing mechanical and hydrodynamic factors determining the meter' error variation.

Since the error of rotary vane meters is determined by liquid leakage through the gaps between rotor vanes and the housing, numerical simulation is based on wellknown equations describing flow in the channel one wall of which is movable. Such model used by investigators and manufacturers but this is not a simple task since liquid leakage depends on the gap parameters, liquid viscosity, pressure drop in the meter, rotor spin speed, which is directly related to the flow rate, and some other parameters. Therefore, the results of numerical calculation obtained by applying simplified assumptions usually deviate from the results of real flow conditions [1].

\footnotetext{
${ }^{\text {a }}$ Corresponding author: nerijus.pedisius@lei.lt
} 
Useful information on the flow structure in rotary vane meter can be obtained by result analysis of researches related to the flow over the cavity or when a flat surface moves over the cavity where a complicated vortical movement is formed, the structure of which significantly varies considering cavity dimensions, shape and Re number [2]. Usually at low Re, large scale and low frequency structures accumulating the biggest part of the flow energy are observed. With Re increase, high frequency pulsations indicating the occurrence of thinner (smaller) structures are more vivid [3, 4]. Centrifugal forces enable the formation of 3D instabilities. Such investigation results should be estimated in numerical simulations of rotary vane meter performance since liquid leakages from the meter chamber through the gaps as well as meter errors may be strongly related to the structure of the liquid movement in the chambers.

Measurement accuracy of turbine meters under the influence of different factors, including viscosity of liquid or gas is experimentally most investigated issue. The most significant results of these investigations were identification of a universal pattern that links the meter factor with rotation frequency and kinematic viscosity as well as acceptance of $\mathrm{Re}$ number as a distinctive parameter for the process characterization. However, error or calibration curves assumed a universal shape only at high $\mathrm{Re}$ where curves become viscosity independent.

Recently investigation of relationship between measurement errors of turbine meters in liquids and gases of various viscosity and gas still persists. This is of particular importance at low $\mathrm{Re}$ for the considerable influence of mechanical and viscosity drags. A variety of ways for presenting turbine meter calibration results as well as calibration methods are analysed and applied [59]. The construction of new calibration facilities operating at high gas pressure or using alternate test fluids are developing. Beside the mentioned influencing factors and like as for rotary vane meters, liquid or gas leakages in turbine meter also depend on an interaction of meter' blades and casing.

Thus, this study aimed to define physical reasons for variances of error regularities in order to justify possibilities to forecast measurement accuracy of turbine and rotary vane meters under operational conditions in case of significantly changed kinematic viscosity of gas or liquid.

Analysis of relationship between error and Re was conditioned by the still further quite wide application in practice of meters without pulse counters and strong needs to perform monitoring meter' operation and its timely calibration.

\section{Testing procedures}

Rotary vane and turbine meters used for custody transfer were chosen for research on influence of liquid and gas viscosity.

Rotary vane meters DN100 were investigated in four different liquids (diesel fuel, Jet-A1 kerosene, Exxsol
D80 kerosene and petrol) and in on-site conditions (fuel terminals) using portable measurement equipment as well as in the laboratory conditions using stationary facility; meter DN65 - in Exxsol D80 and only in laboratory conditions. Density and kinematic viscosity of the liquids at temperature range (10 to 25$){ }^{\circ} \mathrm{C}$ varied from $719 \mathrm{~kg} / \mathrm{m}^{3}$ to $838 \mathrm{~kg} / \mathrm{m}^{3}$ and from $0.67 \mathrm{~mm}^{2} / \mathrm{s}$ to $5.5 \mathrm{~mm}^{2} / \mathrm{s}$, respectively.

The real volume of the flowing liquid was measured using reference measuring tanks of volumes $0.5 \mathrm{~m}^{3} ; 2.0$ $\mathrm{m}^{3}$ and $5.0 \mathrm{~m}^{3}$, taking into account the flow rate, which changed from $12 \mathrm{~m}^{3} / \mathrm{h}$ to $120 \mathrm{~m}^{3} / \mathrm{h}$. Density and dynamic viscosity of liquid samples were measured using the meter SVM 3000 at temperatures measured during the experiment. Density measurement uncertainty was $0.0005 \mathrm{~g} / \mathrm{cm}^{3}$, viscosity was $0.35 \%$. Best volume measurement capability using volumetric measurement method, taking into account liquid flow rate, varied within range from $0.060 \%$ to $0.065 \%$. Under laboratory conditions at flow rate up to $50 \mathrm{~m}^{3} / \mathrm{h}$, a weighing method was also applied to measure the mass of the flowing liquid, which guaranteed the measurement uncertainty $(0.040$ to 0.045$) \%$. Since in all cases the liquid out flew to the open reference measuring tank or to the reservoir on balances, the influence of the pressure on the measurement results was not estimated.

While measuring air (gas) flow rates at the atmospheric and operational pressures, the reading changes of few types of conventional turbine gas meters were investigated and analyzed in the following order:

- initially, meter' errors at the atmospheric pressure were investigated in the laboratory facility at the air flow rate $(100$ to 9700$) \mathrm{m}^{3} / \mathrm{h}$ and measurement uncertainties $(0.25$ to 0.30$) \%$. International meteorological traceability of reference turbine meters was maintained by calibrating them in the PTB (Germany) national laboratory with uncertainties ( 0.12 to 0.15$) \%$ at the atmospheric pressure and was proved by interlaboratory comparisons;

- after that the meters were calibrated in the laboratories (Pigsar or Karlsruhe, Germany; Force, Denmark) at the pressure (0.61 to 3.5) $\mathrm{MPa}$;

- in high-pressure facility, the calibrated meter was returned to the laboratory, and the actions specified in the first step were performed.

Turbine meters had nominal sizes DN400; 250 and 150 , lengths $1200 ; 750$ and $450 \mathrm{~mm}$. The frontal bearing box with holders in front of the meter' rotor formed an annular channel, heights of which were 56;33.5 and (15.5 to 17.5 ) $\mathrm{mm}$. In two cases for meters DN250, the annular channel did not begin from the flow straightener at the inlet but from the middle of the elongated up to 900 $\mathrm{mm}$ casing. In the other meters, the annular channel began from the flow straightener directly. For each meter air (gas) flow rate varied in the range specified by the meter' manufacturer.

The results of meter' errors variation were analyzed utilizing Reynolds number calculated according to the inlet pipe diameter $(D)$ for rotary vane meter and to the hydraulic diameter of the inlet annular channel $\left(D_{h}=\left(D-D_{i}\right)\right)$ for turbine meter. 


\section{Results and discussions}

\subsection{Influence of liquid viscosity on measurement errors of rotary vane meters}

The experimental results of errors dependence on $\mathrm{Re}_{\mathrm{D}}$ are shown in Figure 1. It can be observed that the error curves have the denominated peak, up to which the errors are strongly stratified due to the influence of mechanical friction and hydrodynamic forces induced by liquid viscosity.

The errors decrease with the increase of the liquid flow rate and $R e_{D}$. This is the consequence of increased rotating forces, due to which the influence of mechanical friction and retarding viscosity forces relatively decreases until the equilibrium of rotating and retarding forces is reached. The peak of the errors moves towards higher flow rates when the viscosity decreases. There is evidence to state that at high liquid viscosity there is also the peak of error curve, but it reveals itself with decreasing $\operatorname{Re}_{\mathrm{D}}$.

Comparison of errors and pressure loss curves shows that the peak of errors correlates with the point where the sharp change of pressure loss curve occurs. It means that analogous regimes are inherent for flow in the rotary vane meter passage, as for the flow in the channel. In the range of low $\mathrm{Re}_{\mathrm{D}}$, the pressure losses are proportional to the flow velocity, whereas physical parameters of the liquid determine error variation. In the range of high $\mathrm{Re}_{\mathrm{D}}$, due to significantly increasing turbulent viscosity, the pressure losses change proportionally to the velocity square; thus, the ratio of retarding and rotating forces increases with the increase of the flow rate, and the errors start to approach more negative values.

Thus the transition of the flow in the meter' passage to the turbulent flow regime causes the formation of the universal regularity pattern of error variation and its tendencies to get a certain asymptotic value when $R_{D}$ approaches to $10^{6}$. The last phenomenon means that liquid viscosity doesn't influence more flow pattern and the equilibrium of rotating and retarding forces has steadied. Difference between error curves (2) and (3) in Figure 3 is more related to different meter sizes than to liquid viscosity values. Something more about it will be presented in the next section

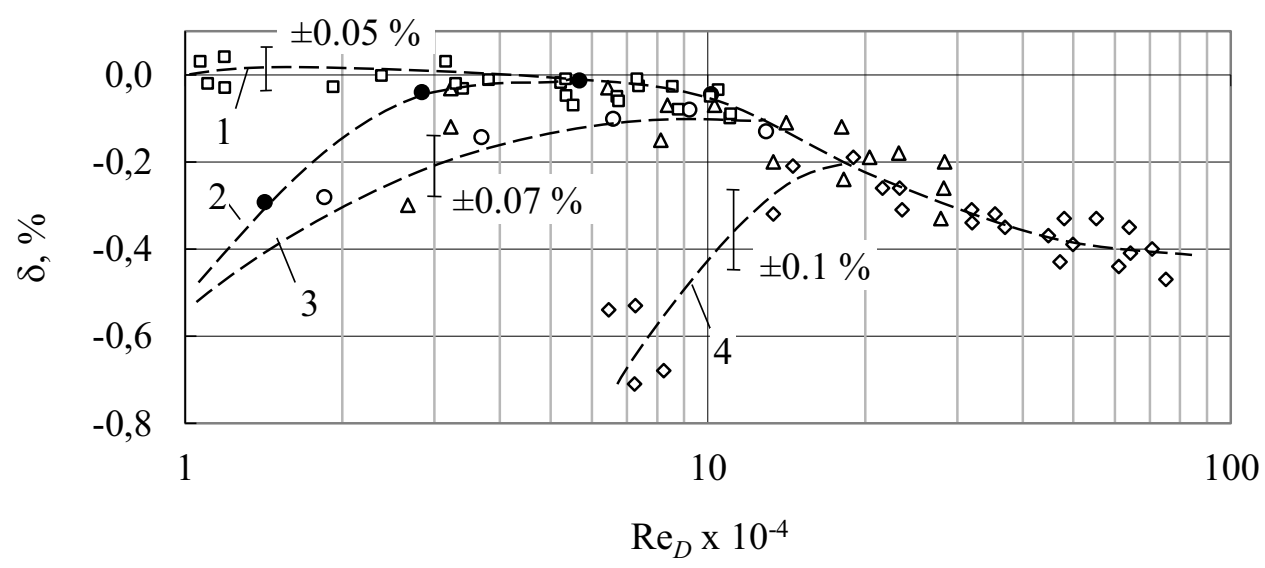

Figure 1. Rotary vane meter' errors variation versus Re $\mathrm{D}_{\mathrm{D}}$ meter DN100: 1 - diesel fuel $\left(\bar{v}=4.6 \mathrm{~mm}^{2} / \mathrm{s}\right) ; 3-\mathrm{Exxsol}_{\mathrm{D} 80}$ and aviation kerosene $\left(\bar{v}=(2 \pm 0.3) \mathrm{mm}^{2} / \mathrm{s}\right) ; 4-$ petrol $\left(\bar{v}=0.73 \mathrm{~mm}^{2} / \mathrm{s}\right)$; meter DN65: $2-$ Exxsol D80 kerosene $(\bar{v}=$ $2.3 \mathrm{~mm}^{2} / \mathrm{s}$ )

\subsection{Influence of air (gas) viscosity on measurement errors of turbine meters}

The results of variation of turbine meter' errors at the atmospheric pressure presented in Figure 2 show the more complicated its distribution which is primarily predetermined by the different designs and sizes of meters selected for investigation. Furthermore, at low $\mathrm{Re}_{\mathrm{Dh}}$ that corresponds the meter' operation or calibration conditions at atmospheric pressure, the character of the error variation is strongly influenced by the substantial retarding non-viscous and viscous forces, relatively small rotating torque, the geometry of the annular channel and rotor, specific features of the transitional flow in the annular channel and some other factors applied for linearization of calibration curve.
The considerable dispersion of the turbine meter' errors from $-1.5 \%$ to $3 \%$ at the atmospheric pressure and low $\mathrm{Re}_{\mathrm{Dh}}$ is observed. However, this dispersion reduces up to $\pm 0.5 \%$ or even less, when $\operatorname{Re}_{\mathrm{Dh}}$ increases up to $\sim 4 \cdot 10^{5}$. It can be briefly summarized that, as in the case of rotary vane meter, the shift of $\mathrm{Re}_{\mathrm{Dh}}$ to the higher value diminishes the influence of factors significant at lower $\mathrm{Re}_{\mathrm{Dh}}$ and equalizes the impact of remaining factors when high $\mathrm{Re}_{\mathrm{Dh}}$ is reached.

At first sight, the error dispersion in Figure 2 seems to be chaotic, but it is not so if we take a look more carefully. Thereto in Figure 3, these results are presented as normalized values against parameter $\mathrm{Re}_{\mathrm{Dh}} / \mathrm{k}$ :

$$
\Delta=\frac{\left(\delta-\delta_{\min }\right)}{\left(\delta_{\max }-\delta_{\min }\right)}=f\left(\mathrm{R} e_{D h} / k\right) ;
$$

here $\delta_{\min }, \delta_{\max }-$ minimum and maximum of meter error; $k$-coefficient. 


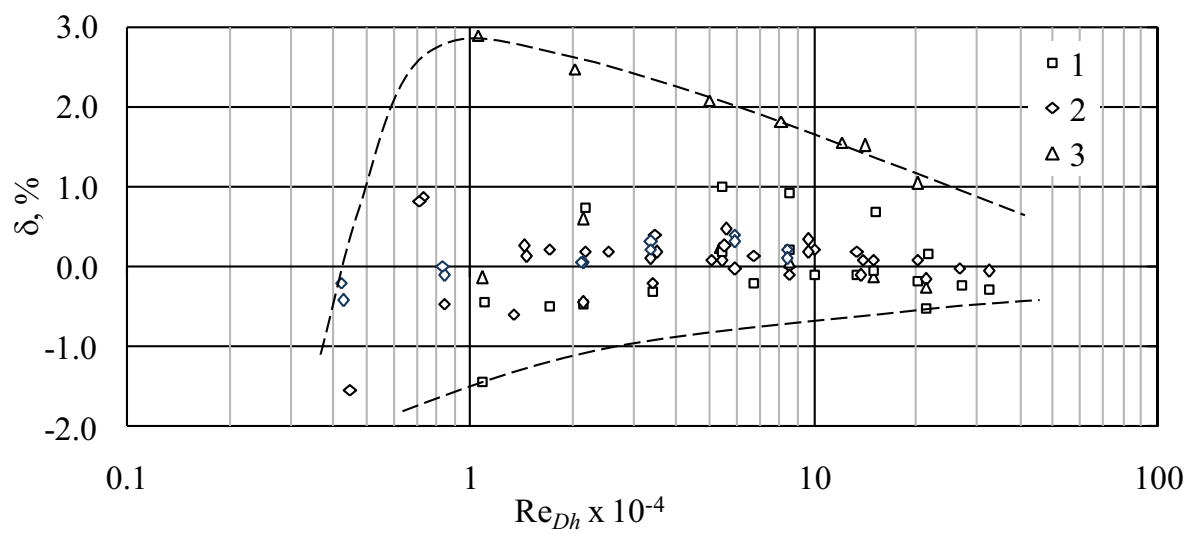

Figure 2. Comparison of error variation of all investigated turbine meters at the atmospheric pressure $\left(\bar{v}=15 \mathrm{~mm}^{2} / \mathrm{s}\right): 1-$ error curves with one prime peak; 2 - with 2 peaks or with non-uniform approach to the prime peak; 3 - without prime peak

Note. Only in this case, coefficient $\mathrm{k}$ is introduced to reach the closeness of the error curves of different meter sizes. Single $\mathrm{Re}_{\mathrm{Dh}}$ does not evaluate the fact that turbine meter is rotating device and rotating torque depends on the height of blades and its distance from the rotor axis as well as on other numerous factors. For the first and partial approximation to more universal curve, it was assumed $\mathrm{k}$ being equal to the distance of the blade center from rotor axis.

Figure 3 shows that it is possible to distinguish at least 3 different shapes of error curves. The first type of error curves (1) has one prime peak located in the region of $\mathrm{Re}_{\mathrm{Dh}} / \mathrm{k}=(5$ to 8$) \cdot 10^{5} \mathrm{~m}^{-1}$. As in the case of rotary vane meter, prime peak divides meter operation interval into two parts at the first of which meter operates at low $\mathrm{Re}_{\mathrm{Dh}}$ and it is influenced by the all factors mentioned above. At $\mathrm{Re}_{\mathrm{Dh}} / \mathrm{k}>8 \cdot 10^{5}$ meter begins to operate in turbulent flow regime independent on fluid viscosity as well as on mechanical factors and error curves assume universal shape.

The second type of error curves (2) has the first peak located in the region of $\mathrm{Re}_{\mathrm{Dh}} / \mathrm{k} \leq 2 \cdot 10^{5}$.It is possible to make presumption, that in such cases the specific measures are applied for reduction of non-viscous and viscous retarding forces and for increase of rotating torque due to the peculiarities of velocity distribution in the annular channel, flow pulsations typical for early transitional flow regime and ones associated with the phenomena of flow separation and resonance while flowing around the rotating impeller. Under the influence of such measures the error peak is reached faster and it shifts at lower $\mathrm{Re}_{\mathrm{Dh}} / \mathrm{k}$. Since this happens in the area of low $\mathrm{Re}_{\mathrm{Dh}} / \mathrm{k}$, the flow cannot be considered a fully developed turbulent flow. The wavy error variation until the prime error peak demonstrates the flow sensitivity to various disturbances. The prime error peak may form or it can be not clearly visible if the flow is highly disturbed.

The third type of error curves (3) represents the last mentioned conditions when error sustains its variation pattern being typical for turbulent flow regime till prime peak zone is reached.

It is also important to notice that the turning-point of all curves are related with essential changes of rotating and retarding forces ratio as well as errors and located in well-defined zones: prime peak at $\mathrm{Re}_{\mathrm{Dh}} / \mathrm{k}=(5$ to 8$) \cdot 10^{5}$; first peak or minimum at (1to 3$) \cdot 10^{5}$. The slope of error curves between the turning-points is also quite informative. In accordance with such analysis, it is possible to predict the meter operation regimes and effects of meter installation into enlarged pressure air (gas) flow.

Comparison of relationships between the turbine meter errors and $\mathrm{Re}_{\mathrm{Dh}}$ values at the atmospheric and enlarged pressures is presented in Figure 4. As it is seen, in enlarged pressure flow turbine meter' errors remain at the level, to which ones were approached at atmospheric pressure and $\operatorname{Re}_{\mathrm{Dh}} \sim 4 \cdot 10^{5}$ (see Figure 2).

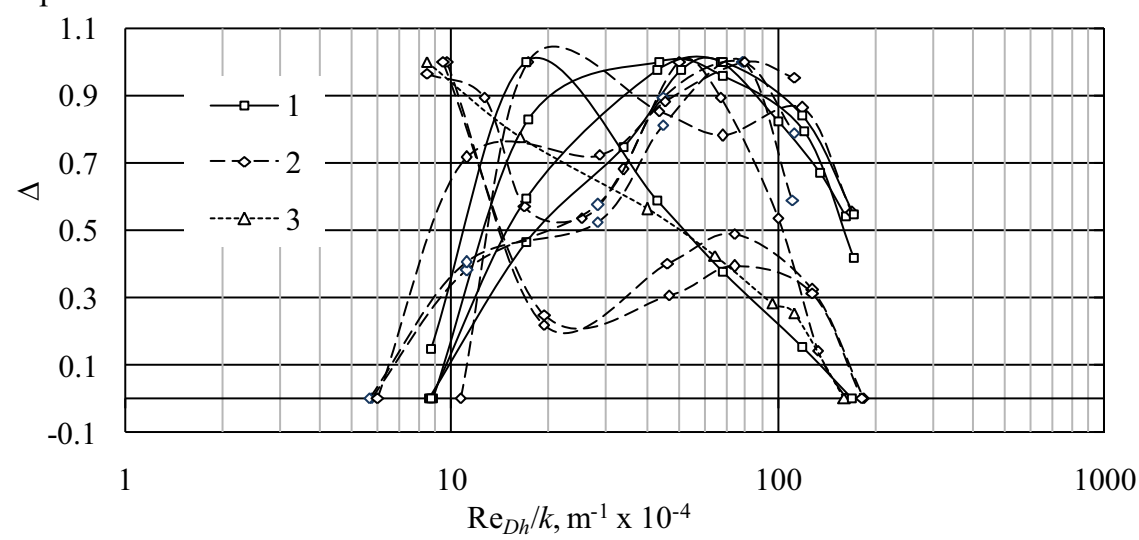

Figure 3. Comparison of normalized error curves of turbine meters: 1 - error curves with one prime peak; 2 - with 2 peaks or with non-uniform approach to the prime peak; 3 - without prime peak 


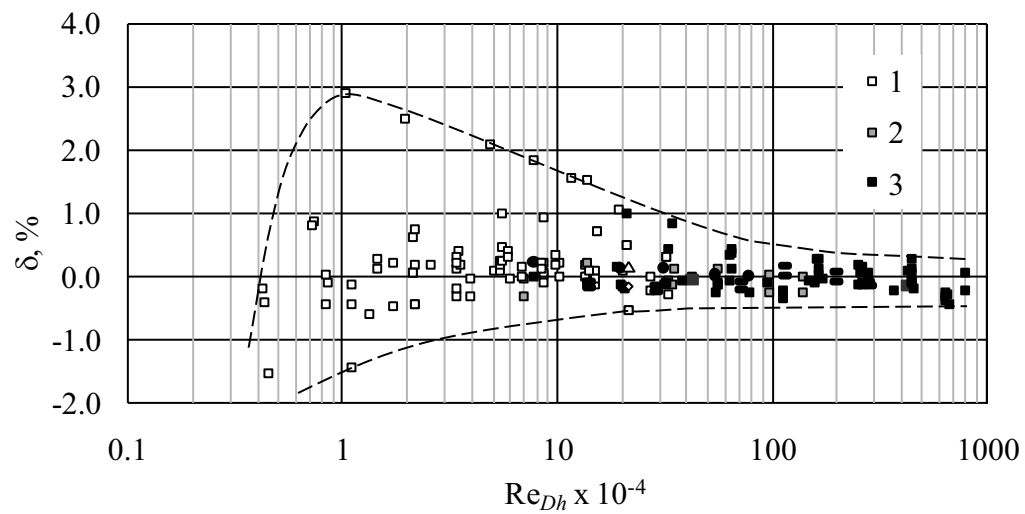

Figure 4. Comparison of error variation of investigated turbine meters at the atmospheric and enlarged pressures: 1 - atmospheric pressure, $\bar{v}=15 \mathrm{~mm}^{2} / \mathrm{s} ; 2$ - pressure $0.61 \mathrm{MPa}, \bar{v}=2.74 \mathrm{~mm}^{2} / \mathrm{s} ; 3$ - pressure $3.5 \mathrm{MPa}, \bar{v}=0.46 \mathrm{~mm}^{2} / \mathrm{s}$

It means that with an increase of pressure, the greatest error variations occur in the area of low $\mathrm{Re}_{\mathrm{Dh}}$ as the smallest negative error values due to the increase of density and rotary torque always have to shift toward positive values, while peak value has to decrease because with the increase of $\mathrm{Re}_{\mathrm{Dh}}$ turbulent flow regime is achieved faster, and during it the error begins to change toward negative values due to growing retarding forces

However, if the character of the error variation at the atmospheric pressure is like at turbulent flow regime, the same tendency will remain at the enlarged pressure. This is clearly evident from Figure 5. The similarity principle according to $\mathrm{Re}_{\mathrm{Dh}}$ comes into force. Dashed lines mark the position of forecasted first error peak. The set of the upper points (Figures 4 and $5 b$ ) is attributed to the turbulent flow and demonstrates the limit until which the error peak could maximally shift at low $\mathrm{Re}_{\mathrm{Dh}}$.
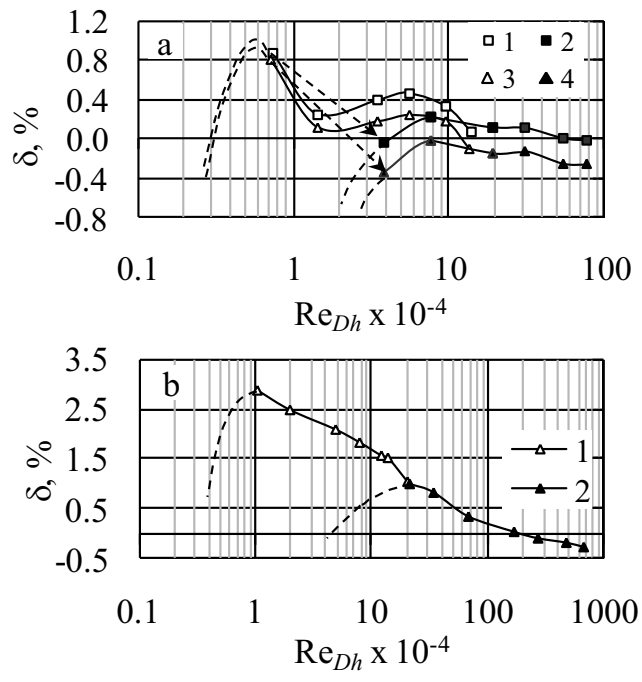

Figure 5. Variation of errors of different type and size turbine meters taking into consideration ReDh: a) - DN 150, conventional type: $1 ; 3$-atmospheric pressure; 2; 4 - pressure $0.61 \mathrm{MPa}$; b) -DN 250, elongated casing: 1 - atmospheric pressure; 2 - pressure $3.48 \mathrm{MPa}$

Finally, it may be concluded that in accordance with the shape of the turbine meter'error curve at the atmospheric pressure, the errors at enlarged pressure can be evaluated with accuracy being tolerable for some practical conclusions. To ensure the reliability of such forecast, it is necessary to analyze the error curve for the widest $\mathrm{Re}_{\mathrm{Dh}}$ range possible at the atmospheric pressure. It is very important to know the shape of the error curve at low $\operatorname{Re}_{\mathrm{Dh}}$ and also at $\mathrm{Re}_{\mathrm{Dh}}>10^{5}$ when the flow becomes stably turbulent and the errors start to approach the constant asymptotic value.

These results are in good agreement with the relationship between meter' factor $\mathrm{K}$ and parameter $(\mathrm{Hz} / \bar{v})[5]$.

\subsection{Comparison of measurement errors of rotary vane and turbine meters}

Figure 6 demonstrates comparison of rotary vane meter (a) and turbine meter (b) measurement error variations in liquid and air (gas) flows under the influence of variable viscosity. For comparison purposes, turbine meter' errors are provided taking into account $\mathrm{Re}_{\mathrm{Dh}}$ since, as indicated above, the flow in a turbine meter is similar to the flow in an annular channel. For the rotary vane meter, inlet diameter (D) was left as the reference parameter.

Despite the different measurement principles between turbine meters and rotary vane meters, analysis of error dependency on Re shows that in both cases these dependencies are very similar. Certain discrepancies according to Re can be explained by inadequate selection of the characteristic geometric parameter due to rather complicated geometry of meter' passage as well as peculiarities of interaction mechanism between the rotors and the casing walls that requires more detailed investigations.

However, the last facts suggest that in highly turbulent flow the impacts of flow separation phenomena and centrifugal forces are also unified, while the flow near the wall isn't considerably influenced by the large scale of turbulent disturbances.

\footnotetext{
${ }^{\mathrm{a}}$ Corresponding author: nerijus.pedisius@lei.lt
} 

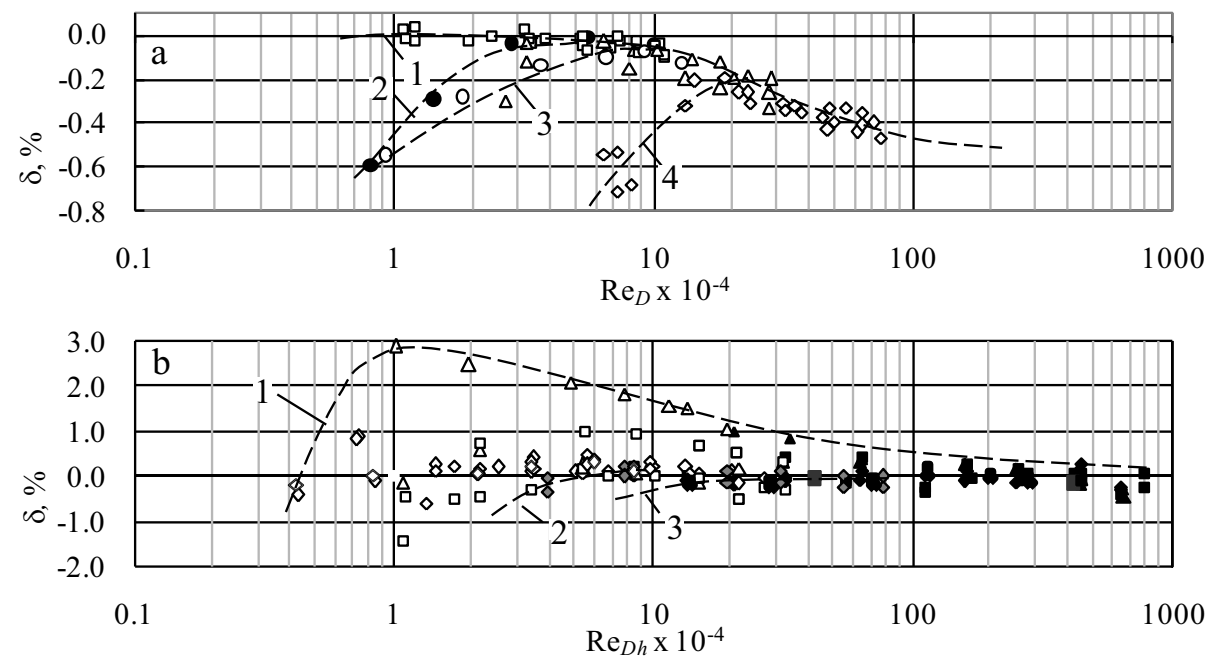

Figure 6. Comparison of error curves of rotary vane and turbine meters considering Red and Re $e_{\text {Dh: }}$ a) - rotary vane meter: $1 ; 2 ; 3$ and 4 - notation is the same as in Figure 1; b) - turbine meter: 1;2 and 3 - notation is the same as in Figure 4

\section{Conclusions}

The present experimental investigation results confirm that the changes of measurement errors of rotary vane and turbine meters are determined by analogous physical factors, and Reynolds similarity principles can be applied to summarise the both type meters calibration results.

Analysis of error curves gives the useful information about the error dependence on the ratio of rotating and retarding forces in all operation range of meter. Prime peak of error curves divides meter operation interval into two parts at the first of which meter operates at low Re and it is strongly influenced by the substantial retarding non-viscous and viscous forces being dependent on meter - construction, specific features of transitional flow regime as well as some other factors, for example, applied for linearization of calibration curve. At high Re, meters start to operate in turbulent flow regime independent on fluid viscosity as well as on mechanical factors and error curves assume universal shapes with tendencies to get a certain asymptotic value when $\mathrm{Re}$ approaches to $10^{6}$.

The determined error variance of mechanical meters can be applied in practice to foresee:

- gas flow rate measurement errors of turbine meters under enlarged pressure when the nature of error variance under atmospheric pressure is known;

- liquid flow rate measurement errors of rotary vane meters when results of error measurement in one liquid are known, and justify an introduction of corrections to evaluate operation conditions.

\section{Acknowledgments}

The authors would like to express their gratitude to JSC "Lithuanian gas" for the opportunity to use the turbine meter calibration results obtained in foreign laboratories at high pressure facilities.

\section{References}

1. E. Von Lavante, S. Poggel, H. Kaya, M. Franz, Numerical Simulation of Flow in Rotor-Casing Gap of a Rotary Piston Flow Meter, Proc. of 14th Int. Flow Measurement Conf., (2010).

2. P. N.Shankar, M. D. Deshpande, Fluid Mechanics in the Driven Cavity, Annu. Rev. Fluid Mech., pp. 93-136, (2000)

3. S. Albensoeder, H. C. Kuhlmann, H. J. Rath, Three-Dimensional Centrifugal-Flow Instabilities in the Lid-Driven-Cavity Problem, Phys. Fluids, 13, 121, (2001)

4. A. K. Prassad, J. R. Koseff, Reynolds Number and End-Wall Effects on a Lid-Driven Cavity Flow, Phys. Fluids, A 1, 208, (1989)

5. A. Ranseth, Reynolds Number Calibration of Turbine Meters, TransCanada calibrations, www.cga.ca/events/documents/ransethalan.pdf.

6. J. G. Pope, J, D. Wriht, A. N. Johnson, M. R. Moldover Extended Lee Model for the Turbine Meter and Calibration With Surrogate Fluids, Flow meas. and instr., 24, pp. 71-82, (2012)

7. P.W. Tang, High-Pressure Calibration of Gas Turbine Meters Using the Reynolds Number Methods, http://www.asgmt.com/default/papers/asgmt 200 9/docs $/ 050 . p d f$.

8. W. F. Z. Lee, H. J. Evans, Density Effects and Reynolds Number Effect on Gas Turbine Flowmeters, J. Basic Engineering, 87, 4, pp. 1043-1052, (1965)

9. S. De Jong, P. M. A. Kam, High Pressure Recalibration of Turbine Meters in Flow Measurement, Proc. of Flomeko'93, ISBN898454-0180-6, (1993)

\footnotetext{
${ }^{\mathrm{a}}$ Corresponding author: nerijus.pedisius@lei.lt
} 